\title{
26th Wilhelm Bernhard Workshop on the Cell Nucleus 20-24 May 2019
}

\section{Dear Participants,}

Welcome to the 26th Wilhelm Bernhard Workshop on the Cell Nucleus. This meeting is jointly organized by the International and the Local Organizing Committees. We have worked together to make this conference informative and enjoyable. We are looking forward to many excellent presentations and discussions during this exciting week in Dijon that will allow all of us to consider new hypotheses and findings, present new data and ideas, establish new collaborations. In addition to an outstanding scientific program, we hope you will enjoy the magnificent setting of Dijon, the former capital of Duchy of Burgundy, a gem of medieval Europe, and currently, one of the centers of French culture, excellent wines and gourmet cuisine recognized by UNESCO as Intangible Cultural Heritage of Humanity.

During the meeting we will discuss a wide spectrum of topics including recent data on structure and functions of various nuclear compartments, nuclear envelope, 3D genome organization, regulation of eukaryotic gene expression, DNA replication and repair, noncoding RNA, cell imaging and dynamics, mechanisms of laminopathies and oncogenesis, and many other topics. Plenary speakers and speakers selected from submitted abstracts, as well as session chairs will provide a framework for discussion. This will allow all participants to evaluate recent achievements in this field of research from different perspectives and to be involved in the development of new directions and approaches, as well as discussing new ways of solving existing problems and difficulties. As usual, Wilhelm Bernhard Workshop provides a platform for opinion exchange between researchers from different countries including Europe, Asia and North America. The special emphasis will be placed on creating a comfortable environment for the communication between established scientists and young researchers and students.

We hope you will enjoy the conference and we hope that plenary sessions, short talks, poster presentations and after-the-meeting discussions will stimulate a breakthrough in our understanding of nuclear structure and functions, as well as the role of nuclear components in pathological conditions.

Oleg Demidov,

Chairman of the Wilhelm Bernhard International Committee 